\title{
Estimación de la superficie cultivada de Palma Aceitera mediante interpretación visual de imágenes satelitales durante los años 2016 - 2020
}

\author{
Osmar Yupanqui Carrasco ${ }^{1}$, Juan Ruben Julca Torres ${ }^{2}$ y Erick Valerio Candia ${ }^{3}$
}

Recibido: 30 mayo 2021 | Aceptado: 7 noviembre 2021

\section{RESUMEN}

Actualmente en el Perú no existe una cifra oficial sobre la cantidad exacta de hectáreas de palma aceitera (Elaeis guineensis Jacq.), por ello, este trabajo apunta a generar información sobre el aumento del cultivo desde el año 2016 hasta el 2020, en las regiones de Ucayali, San Martín, Huánuco y Loreto, mediante la interpretación visual de las imágenes satelitales bianuales y mensuales pantropicales de Planet NICFI, junto con el uso del software QGIS 3.10.2 y la extensión (plugin) Planet. En total, se encontraron 85122,95 ha en el año 2016; 86 866,18 ha en el año 2017; 91 357,80 ha en el año 2018; 94 656,40 ha en el año 2019 y 95 048,37 ha en el año 2020 en las 4 regiones estudiadas, siendo Ucayali la región con mayor cantidad de hectáreas de palma aceitera, con 44 077,82 ha para el año 2020, seguida de San Martín con 35 151,74 ha, Loreto con 13568,94 ha y Huánuco con 2249,87 ha. También se generó la información cartográfica sobre las 16 plantas de procesamiento de palma aceitera encontradas y finalmente, estos resultados se llevaron a una aplicación en Google Earth Engine para su divulgación.

Palabras clave: Interpretación visual, imágenes satelitales, monitoreo, aplicativo.

\footnotetext{
'Bachiller en Ing. Forestal, email: osmar30asis@gmail.com

${ }^{2}$ Egresado de Ing. Forestal, email: jjulcatorres@gmail.com

${ }^{3}$ Estudiante de Ing. Forestal, email: erick.valerio.candia.28@gmail.com
} 


\section{ABSTRACT}

Currently, in Peru there is no official number of the exact amount of hectares of palm oil (Elaeis guineensis Jacq.), thus, this work aims to generate information about the increment of this crop since the year 2016 to the 2020, in the regions of Ucayali, San Martín, Huánuco and Loreto, through visual interpretation of the biannual and monthly pantropical satellite imagery of Planet NICFI, together with the use of the software QGIS 3.10.2 and the plugin Planet. In total, 85122,95 ha were found in the year 2016, 86866,18 ha in 2017, 91357,80 ha in 2018, 94 656,40 in 2019 and 95048,37 in 2020 in the 4 regions studied, being the region with the most amount of hectares of palm oil Ucayali, with 44077,82 ha for the year 2020, followed by San Martín with 35 151,74 ha, Loreto with 13 568,94 ha and Huánuco with 2 249,87, ha. The cartographic information was also generated on the 16 oil palm processing plants found and finally, these results were taken to an application in Google Earth Engine for it's disclosure.

Key words: Visual interpretation, satellite imagery, monitoring, app.

\section{INTRODUCCIÓN}

Según MINAGRI (2012), citado por Leveau (2018), la palma aceitera (Elaeis guineensis Jacq.), es uno de los cultivos oleaginosos de mayor crecimiento registrado a nivel mundial, debido principalmente a su alto poder productivo; es decir, a su mayor rendimiento de tonelada de aceite por hectárea (t/ha), su producción inicia a los 3 años de sembrado y produce comercialmente durante 25 años. Este alto rendimiento se visualiza en la Figura 1.

\section{Oil yield by crop type, 2018}

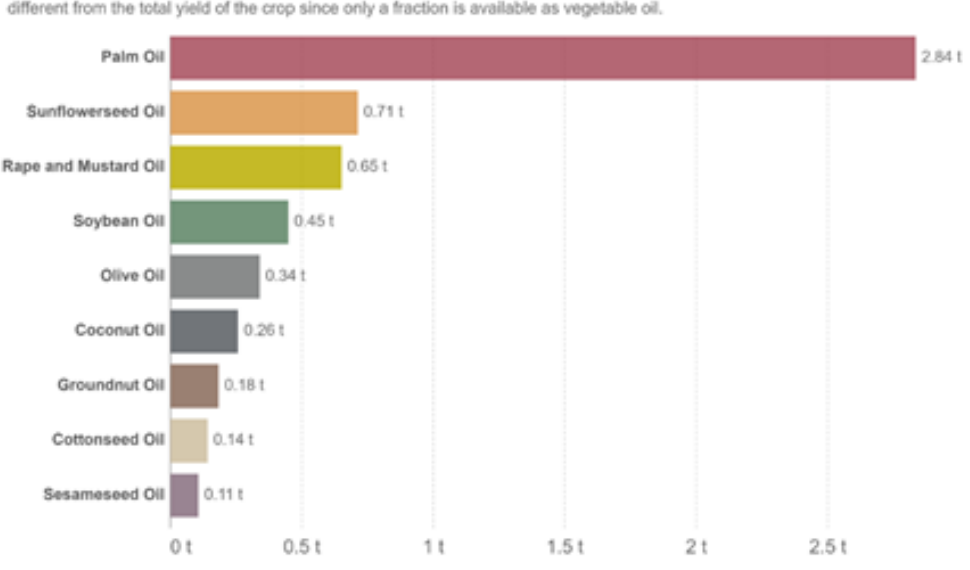

Figura 1.

Rendimiento global de aceites por cultivo y por hectárea. Elaborado por: ourworldindata.org. Fuente: FAOSTAT, 2018. 
Kongsgager \& Reenberg (2012) mencionan que este cultivo está presente en la mayoría de los países en los trópicos, y que ha sido usado históricamente como alimento y medicina. El desarrollo de la industria de la palma aceitera como plantación inició en el sudeste asiático y se ha convertido en uno de los cultivos industriales más importantes en países como Malasia, Indonesia y Tailandia. Sin embargo, la escasez de tierras en estas regiones genera que la industria busque otras regiones para desarrollarse, lo cual explica en parte su crecimiento en África y América Latina (Butler y Laurence, 2009)."

El Perú es un actor pequeño en el mercado mundial de aceite de palma, pero enfrenta muchos de los desafíos ambientales y sociales que se ven en los países productores de palma más grandes, como Indonesia, Malasia, Tailandia y Colombia, algunos de estos desafíos incluyen el control de la deforestación impulsada por la expansión del cultivo de palma aceitera y la violación de los derechos humanos y laborales (SPDE, 2019).

Dammert (2014) afirma que: “A diferencia de sus países vecinos, el Perú no cuenta con otras áreas de bosques húmedos tropicales más allá de los de la cuenca amazónica. Los factores agroecológicos hacen que algunos cultivos, como la palma aceitera, solo crezcan en determinadas zonas de esta región".

Históricamente, los hitos más importantes en la expansión del cultivo de palma aceitera han sido los años 2000 y 2010. Según Sáenz (2017), desde 1970 hasta el 2000 se cultivaron 14667 ha, esto quiere decir que el avance se dio lentamente, mientras que en 2010 contabilizaron 50200 ha, hubo un crecimiento acelerado por la política de sustitución de cultivos ilícitos que el Estado impulsó y la propuesta de cooperación internacional como solución al conflicto social que atravesaba el país.

Oficialmente no hay cifras que indiquen la extensión que posee el cultivo de palma aceitera en el Perú, la Junta Nacional de Palma Aceitera del Perú (JUNPALMA), reporta que hubieron 77537 ha cultivadas de palma aceitera en la Amazonía Peruana para el 2014 (Sáenz, 2017), mientras que Vijay et al. (2018) estima 84500 ha entre el 2010 y 2015, Food and Agriculture Organization (FAO, 2018) reporta para ese mismo año 43 100 ha de palma aceitera, por otra parte, Monitoring of the Andean Amazon Project (MAAP) documenta más de 86600 ha para el 2018 .

La distribución espacial y extensión de la palma aceitera guarda estrecha relación con la distancia existente entre el cultivo y la planta extractora o de procesamiento de aceite de palma aceitera, pues mientras menor sea esta distancia, mayor será la ventaja competitiva que tendrá la empresa dueña del terreno, Angulo, et al. (2017) recomiendan tener una planta de extracción ubicada en un radio no mayor de $35 \mathrm{~km}$, pues la distancia promedio para el abastecimiento de las plantas productoras supera los $100 \mathrm{~km}$ de distancia.

Ante esta situación, el presente estudio actualizará la información histórica sobre la extensión del cultivo de palma aceitera y la ubicación de sus 
plantas de procesamiento en las regiones de Ucayali, San Martín, Huánuco y Loreto, para mejorar el conocimiento de la extensión de este cultivo, y que esta información permita a los tomadores de decisiones entender mejor las dinámicas del cultivo y plantear estrategias para su monitoreo, mediante el desarrollo de un visor de palma aceitera.

\section{MATERIALES Y MÉTODOS}

\section{Área de estudio:}

El presente estudio se centró en los departamentos de Ucayali, San Martín, Huánuco y Loreto, desde el año 2016 hasta el 2020. Se analizaron todas las provincias y distritos de los departamentos mencionados, a fin de cuantificar las hectáreas del cultivo de palma aceitera.

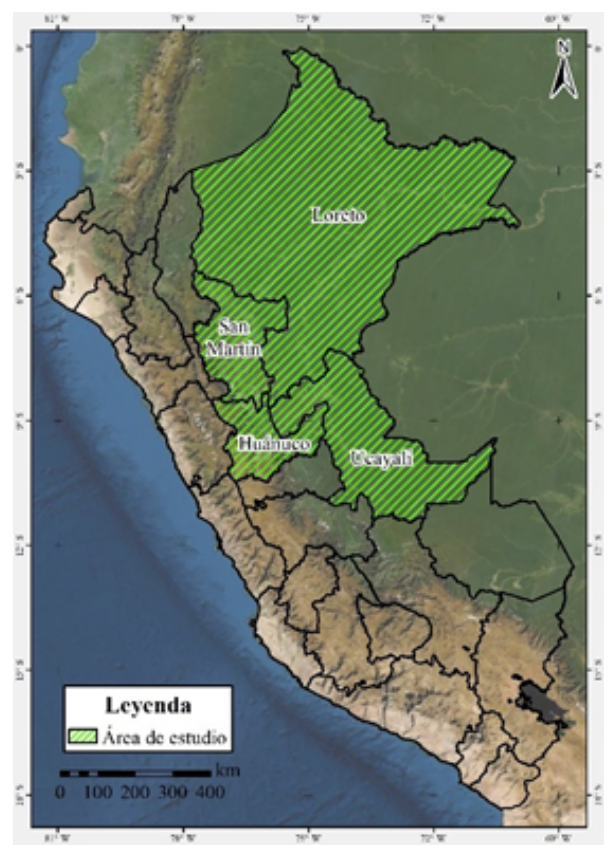

Figura 2.

Mapa base del área de estudio. Elaboración propia.

\section{Materiales:}

Para el desarrollo del estudio se empleó el software de sistemas de información geográfica QGIS 3.10.2 (QGIS.org, 2021), debido a que posee una extensión llamada Planet, que permite acceder libremente a los mosaicos bianuales y mensuales pantropicales, correspondientes a las imágenes de Planet NICFI, que poseen 4,77 $\mathrm{m}$ de resolución espacial, y ya vienen normalizadas, con correcciones atmosféricas y con máscara de nubes (Planet, 2021). También se contó con el mapa base palma aceitera para el 2017 desarrollado por SPDE (2019) y los mapas bases presentes en el mismo software de QGIS, como Bing Aerials y Google Satellite de Google Earth. Otros insumos que permitieron el desarrollo de esta investigación incluyeron la cartografía de palma aceitera entregada por Víctor Galarreta (JUNPALMA) para el 2018 del departamento de San Martín y los resultados de la metodología HCSA/HCV (High Carbon Stock Approach / High Conservation Value) en Tocache, San Martín para el año 2018, otorgados por Richard Vaca de Earthworm Foundation. También se empleó la información cartográfica proveniente del Ministerio de Transportes y Comunicaciones (2019), para la visualización de redes viales, Autoridad Nacional del Agua (2018), para la red hidrográfica y el mapa base de palma aceitera del año 2017 elaborado por SPDE (2019).

\section{Métodos:}

La interpretación de imágenes digitales es básicamente posible de 
dos maneras, generalmente denominadas interpretación visual e interpretación por computadora (E. P. Green et al., 1998). En este estudio el método utilizado para la identificación de áreas de palma aceitera fue la interpretación visual, la cual consiste en la interpretación de imágenes aéreas y satelitales cuyo objetivo es obtener información sobre los objetos y el paisaje. Es un proceso específico de estudio de la realidad geográfica basado en la detección, identificación y localización espacial de objetos individuales y formas del terreno capturados de imágenes de satélite (Lauermann, 2010, como se citó en Svatonova, 2016). La información recopilada de las imágenes está codificada en varios tonos y texturas. Rubín de Celis (2020) nos menciona la habilidad que tienen los seres humanos para relacionar los tonos, colores y patrones espaciales que aparecen en una imagen con los elementos del mundo real, siendo las características evaluadas para su identificación las siguientes:

Siendo los puntos a considerar bajo interpretación propia los siguientes:

a.- Textura: Mayoritariamente lisas.

b.- Color: Representado por una tonalidad de verde oscuro para el cultivo y a veces verde claro.

c.- Forma: Rectangulares, cuadradas o figuras geométricas, con ángulos rectos en su mayoría.

d.- Patrón: Parcelas agrupadas y con cercanía a una planta de procesamiento de aceite de palma.

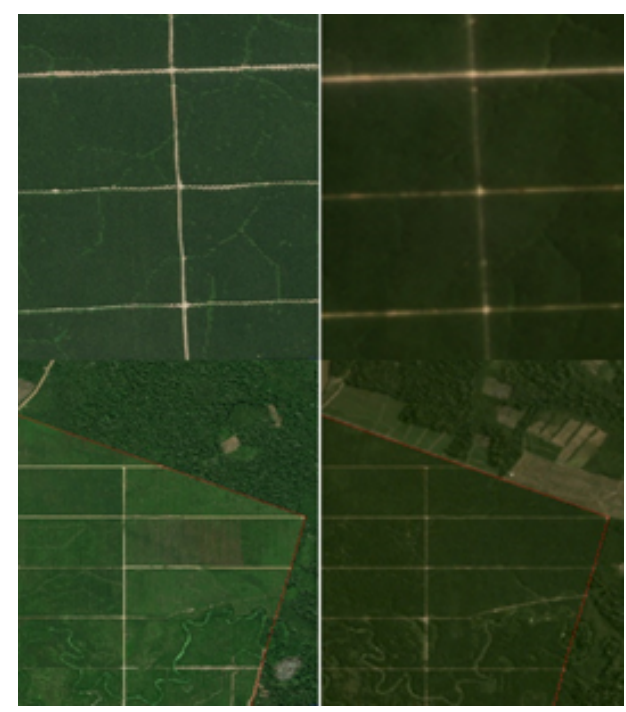

Figura 3.

Comparación de imágenes Bing (izquierda) de muy alta resolución y Planet NICFI 2020 (a la derecha) de menor resolución.

Elaboración propia.

Una vez identificados los parámetros anteriormente mencionados, se procedió con la digitalización de los polígonos para la línea histórica de palma aceitera del 2016 y continuando con los años consecutivos hasta el 2020 empleando el software de sistemas de información geográfica QGIS 3.10.2, también se verificó que no haya un traslape espacial entre las capas mediante el empleo de herramientas topológicas. Finalmente, se contabilizaron las hectáreas de palma anuales por departamento, provincia y distrito mediante el corte de la capa generada, con las capas de límites departamentales, provinciales y distritales del Instituto Geográfico Nacional (IGN). 
Para la identificación de las plantas de procesamiento de palma aceitera, se empleó la información otorgada por JUNPALMA, que otorga referencias de ubicación de las diferentes refinerías de las 4 regiones mencionadas.

Toda la información cartográfica generada se llevó a la plataforma de Google Earth Engine (Gorelick et al., 2017), que emplea el lenguaje de programación de Javascript, donde se desarrolló un aplicativo para que el público en general pueda acceder a la información, además de añadir herramientas adicionales para controlar la opacidad de las capas y barras de desplazamiento a las plantas de procesamiento y a los polígonos más grandes de palma aceitera, también se programó la plataforma para que al hacer clic en una parcela, se muestre el área del polígono y también las coordenadas (longitud y latitud).

\section{RESULTADOS}

\section{Línea Base 2016}

Para la línea base del año 2016 se encontró un total de 85 122, 95 ha, siendo la región de Ucayali la que contó con mayor cantidad de hectáreas de palma aceitera, con 38 808,92 ha, seguido de San Martín con 31798,93 ha, Loreto con 12 985,13 ha y Huánuco con 1529,97 ha.

A nivel provincial, las mayores superficies (ha) de palma aceitera se encuentran en Tocache (San Martín), Coronel Portillo y Padre Abad (Loreto) con 23 514, 35; 21 134,72 ha

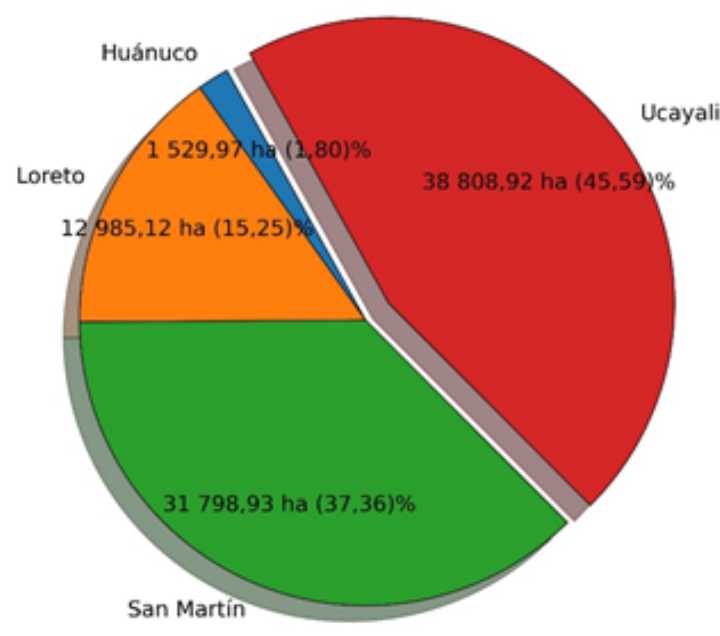

Figura 4.

Superficie cultivada de Palma Aceitera en el área de estudio para la línea base 2016. Elaboración propia.

y 17674,20 ha respectivamente. A nivel distrital se encontró que Tocache (Tocache-San Martín) cuenta con mayor cantidad de área de palma aceitera, con 14818,48 ha, seguido por Yurimaguas (Alto AmazonasLoreto), con 12 728,53 ha, Campo Verde y Nueva Requena (Coronel Portillo - Ucayali), con 10686,82 ha y 10300,65 ha respectivamente.

Incrementos anuales de palma 2017-2020

La Tabla 1 detalla el incremento anual del cultivo de palma aceitera en diferentes niveles: departamental, provincial y distrital, además del total acumulado.

A nivel distrital, para la región de Huánuco, el distrito de Cholón fue el que obtuvo el máximo incremento de cultivo de palma aceitera con 323,85 
ha en el año 2019, mientras que Tournavista fue el que obtuvo el mínimo aumento de cultivos de palma con 2,10 ha en el año 2018. Mientras que, en Loreto, el distrito de Yurimaguas fue el que obtuvo el máximo incremento de cultivo de palma aceitera con 327,61 ha en el año 2018, mientras que Padre Márquez obtuvo el mínimo incremento de cultivo de palma aceitera con 1,96 ha en el año 2018. Por otra parte, en San Martín, el distrito de Papaplaya obtuvo el máximo incremento de cultivo de palma aceitera con 664,17 ha en el 2017, mientras que Nuevo progreso obtuvo el mínimo incremento de cultivo de palma aceitera con 1,85 ha en el 2020. Finalmente, en la región de Ucayali, el distrito de Campoverde obtuvo el máximo incremento de cultivo de palma aceitera con 1273,19 ha en el año
2018, mientras que Callería obtuvo el mínimo incremento de cultivo de palma aceitera con 0,45 ha en el 2018 .

A nivel provincial, Coronel Portillo, en Ucayali obtuvo el máximo incremento de cultivo de palma aceitera con 1395,88 ha en el 2018, mientras que Puerto Inca, en Huánuco obtuvo el mínimo incremento de cultivo de palma aceitera con 3,04 ha en el 2019.

A nivel departamental, San Martín fue el departamento con el mínimo aumento del cultivo de palma aceitera con 53,60 ha en el 2020, mientras que Ucayali fue el que obtuvo el mayor incremento de cultivo de palma aceitera con 2606,23 ha en el 2018 .

Estos incrementos anuales del cultivo de palma aceitera por departamento y por año, se visualizan en la Figura 5.

\begin{tabular}{|c|c|c|c|c|c|c|}
\hline $\begin{array}{l}\text { Departamento/ } \\
\text { Provincia/Distrito }\end{array}$ & $\begin{array}{c}\text { Línea Base } \\
2016\end{array}$ & $\begin{array}{c}\text { Aumento } \\
2017\end{array}$ & $\begin{array}{c}\text { Aumento } \\
2018\end{array}$ & $\begin{array}{c}\text { Aumento } \\
2019\end{array}$ & $\begin{array}{c}\text { Aumento } \\
2020\end{array}$ & $\begin{array}{c}\text { Total } \\
\text { acumulado } \\
\text { (ha) }\end{array}$ \\
\hline Huánuco & 1529,97 & 123,44 & 199,08 & 326,90 & 70,48 & 2249,87 \\
\hline Marañón & 506,37 & 18,22 & 102,61 & 323,85 & & 951,05 \\
\hline Cholón & 506,37 & 18,22 & 102,61 & 323,85 & & 951,05 \\
\hline Puerto Inca & 1023,60 & 105,22 & 96,47 & 3,04 & 70,48 & 1298,82 \\
\hline Honoria & 704,62 & 99,10 & 94,37 & 3,04 & 70,48 & 971,61 \\
\hline Tournavista & 318,98 & 6,12 & 2,10 & & & 327,20 \\
\hline Loreto & 12985,13 & 47,39 & 343,73 & 192,70 & & 13568,94 \\
\hline Alto Amazonas & 12936,20 & 42,39 & 327,61 & 192,70 & & 13498,90 \\
\hline Teniente César López Rojas & 207,67 & & & & & 207,67 \\
\hline Yurimaguas & 12728,53 & 42,39 & 327,61 & 192,70 & & 13291,23 \\
\hline Maynas & 48,92 & 5,00 & 14,15 & & & 68,08 \\
\hline San Juan Bautista & 48,92 & 5,00 & 14,15 & & & 68,08 \\
\hline Ucayali & & & 1,96 & & & 1,96 \\
\hline Padre Márquez & & & 1,96 & & & 1,96 \\
\hline San Martín & 31798,93 & 855,02 & 1342,58 & 1101,60 & 53,60 & 35151,74 \\
\hline Lamas & 7635,82 & 20,06 & 501,07 & 187,90 & & 8344,85 \\
\hline Barranquita & 4044,42 & 9,34 & 121,19 & 45,07 & & 4220,03 \\
\hline Caynarachi & 3591,40 & 10,72 & 379,87 & 142,83 & & 4124,82 \\
\hline
\end{tabular}




\begin{tabular}{|c|c|c|c|c|c|c|}
\hline San Martín & 648,76 & 679,76 & 225,27 & 325,84 & & 1879,63 \\
\hline El Porvenir & 512,02 & 15,60 & 10,30 & 41,51 & & 579,42 \\
\hline Papaplaya & 136,74 & 664,17 & 214,98 & 284,33 & & 1300,20 \\
\hline Tocache & 23514,35 & 155,20 & 616,24 & 587,86 & 53,60 & 24927,26 \\
\hline Nuevo Progreso & 135,74 & 7,86 & 77,62 & & 1,85 & 223,07 \\
\hline Pólvora & 4362,04 & 78,40 & 188,13 & 141,80 & 13,97 & 4784,33 \\
\hline Tocache & 14818,48 & 0,35 & 214,86 & 289,20 & & 15322,89 \\
\hline Uchiza & 4198,09 & 68,60 & 135,63 & 156,87 & 37,78 & 4596,97 \\
\hline Ucayali & 38808,92 & 717,37 & 2606,23 & 1677,41 & 267,88 & 44077,82 \\
\hline Coronel Portillo & 21134,72 & 109,22 & 1395,88 & 741,19 & 83,84 & 23464,85 \\
\hline Callería & 78,68 & & 0,45 & & & 79,13 \\
\hline Campoverde & 10686,82 & 82,56 & 1273,19 & 605,17 & & 12647,74 \\
\hline Nueva Requena & 10300,65 & 26,66 & 71,74 & 119,71 & & 10518,77 \\
\hline Yarinacocha & 68,57 & & 50,49 & 16,31 & 83,84 & 219,21 \\
\hline Padre Abad & 17674,20 & 608,16 & 1210,35 & 936,22 & 184,04 & 20612,97 \\
\hline Alexander Von Humboldt & 122,94 & & 4,13 & 11,46 & 4,58 & 143,11 \\
\hline Curimaná & 4790,80 & 61,98 & 70,49 & 76,90 & 13,55 & 5013,71 \\
\hline Neshuya & 8394,29 & 468,57 & 750,84 & 491,21 & 165,91 & 10270,83 \\
\hline Padre Abad & 4366,17 & 77,61 & 384,90 & 356,65 & & 5185,32 \\
\hline Total (ha) & 85122,95 & 1743,23 & 4491,62 & 3298,60 & 391,97 & 95048,37 \\
\hline Total acumulado (ha) & 85122,95 & 86866,18 & 91357,80 & 94656,40 & 95048,37 & \\
\hline
\end{tabular}

Tabla 1.

Superficie cultivada de palma aceitera a niveles departamentales, provinciales y distritales para la línea base y años consecutivos.

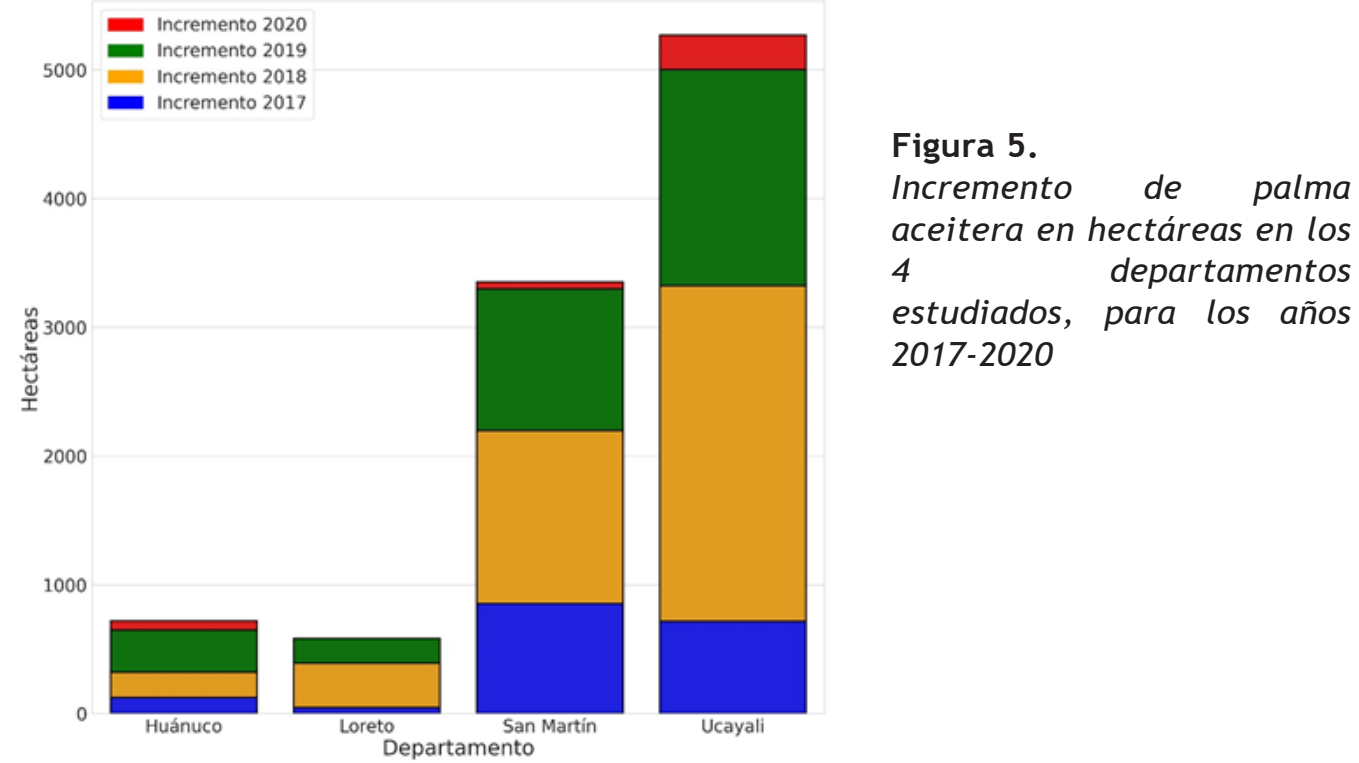


Para el año 2017, la región de Huánuco fue la que obtuvo un mayor incremento porcentual del cultivo de palma aceitera con $8,1 \%$, seguido por San Martín con 2,7\%, Ucayali con $1,8 \%$ y Loreto con $0,4 \%$. En el 2018, la región de Huánuco también fue la que obtuvo un mayor incremento porcentual del cultivo de palma aceitera con $12 \%$, seguido por Ucayali con 6,6 \%, San Martín con $4,1 \%$ y Loreto con $2,6 \%$. Siguiendo la misma tendencia, en el 2019, la región de Huánuco fue la que obtuvo un mayor incremento porcentual de cultivo de palma aceitera con 17,6\%, seguido de Ucayali con 4\%, San Martín con $3,2 \%$ y Loreto con $1,4 \%$. Finalmente, para el 2020, la región de Huánuco fue la que obtuvo un mayor incremento de cultivo de palma aceitera siendo el 3,2\%, seguido de Ucayali con $0,6 \%$ y San Martín con $0,2 \%$, siendo nulo el incremento de Loreto para este año.

La región de Huánuco fue la que obtuvo el mayor incremento porcentual de hectáreas totales en la línea histórica analizada, desde el 2016 (línea base) hasta el 2020, con un $47,1 \%$, seguido por Ucayali con $13,6 \%$, San Martín con 10,5\% y finalmente Loreto con 4,5\%.

\section{Plantas de procesamiento}

En total se encontraron 16 plantas de procesamiento de palma aceitera en las regiones estudiadas, asociadas a diferentes empresas. Se detalla la ubicación política de estas refinerías y sus coordenadas en la Tabla 2.

\begin{tabular}{|c|c|c|c|c|c|c|}
\hline $\mathbf{N}^{\circ}$ & $\begin{array}{c}\text { Nombre de la } \\
\text { empresa }\end{array}$ & Departamento & Provincia & Distrito & $\begin{array}{c}\text { Coordenada } \\
\text { X (UTM) }\end{array}$ & $\begin{array}{c}\text { Coordenada } \\
\text { Y (UTM) }\end{array}$ \\
\hline 1 & OLAMSA-1 & Ucayali & Coronel Portillo & Campoverde & 504583,76 & 9044399,19 \\
\hline 2 & OLAMSA-2 & Ucayali & Coronel Portillo & Campoverde & 518388,61 & 9061156,15 \\
\hline 3 & OLPASA & Ucayali & Padre Abad & Padre Abad & 429102,41 & 8998579,22 \\
\hline 4 & INDOLMASA & Ucayali & Padre Abad & Neshuya & 503253,49 & 9044079,58 \\
\hline 5 & INDEPAL UCAYALI SA & Ucayali & Padre Abad & Neshuya & 501670,16 & 9040960,47 \\
\hline 6 & $\begin{array}{l}\text { AGROPECUARIA } \\
\text { ROSSEL SRL }\end{array}$ & Ucayali & Coronel Portillo & Campoverde & 507183,78 & 9049342,13 \\
\hline 7 & OLPUSAC & Ucayali & Coronel Portillo & Campoverde & 509398,35 & 9050946,83 \\
\hline 8 & $\begin{array}{l}\text { INDUSTRIAS PALM } \\
\text { OLEO SAC }\end{array}$ & Ucayali & Coronel Portillo & Callería & 541127,78 & 9070903,07 \\
\hline 9 & OLNA PERU SAC & Huánuco & Puerto Inca & Honoria & 521914,96 & 9019933,17 \\
\hline 10 & $\begin{array}{l}\text { INDUSTRIAS DEL } \\
\text { SHANUSI SA }\end{array}$ & Loreto & Alto Amazonas & Yurimaguas & 370949,56 & 9322716,55 \\
\hline 11 & INDUPALSA & San Martín & Lamas & Caynarachi & 358516,89 & 9302337,12 \\
\hline 12 & OLPESA & San Martín & Tocache & Pólvora & 324347,57 & 9099735,61 \\
\hline 13 & $\begin{array}{c}\text { INDUSTRIAS DEL } \\
\text { ESPINO SA }\end{array}$ & San Martín & Tocache & Tocache & 335740,16 & 9078384,48 \\
\hline 14 & BIOANDES EIRL & Ucayali & Coronel Portillo & Campoverde & 516231,00 & 9059194,11 \\
\hline
\end{tabular}




\begin{tabular}{ccccccc}
\hline 15 & ASOCIACION & Huánuco & Puerto Inca & Honoria & 528468,07 & 9046024,32 \\
AGROPECUARIA & NUEVO AMANECER & & & & \\
16 & BIODIESEL UCAYALI \\
SRL & Ucayali & Coronel Portillo & Campoverde & 497109,83 & 9067562,78 \\
\hline
\end{tabular}

Tabla 2.

Ubicación de las plantas de procesamiento de palma aceitera.

\section{Visor de palma aceitera}

Se generó el visor de palma aceitera mediante la plataforma de Google Earth Engine, el cual es de libre acceso y se puede acceder con el siguiente enlace: https://osmaryupanqui.users.earthengine.app/view/palm-oil-spde

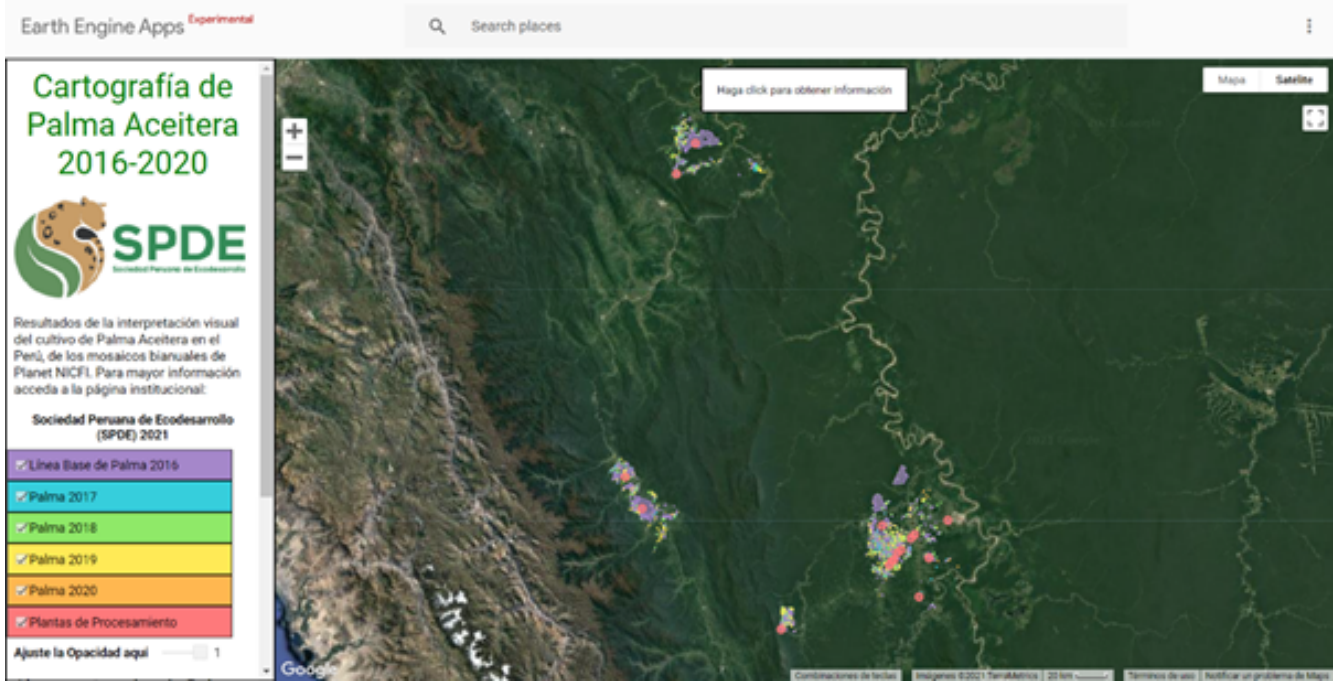

Figura 6.

Distribución espacial del cultivo de Palma Aceitera en el visor creado en Google Earth Engine. Elaboración propia.

\section{DISCUSIONES}

OXFAM (2008), citado por Leveau R. (2018), estimó las áreas altamente adaptables y moderadamente adaptables para el cultivo de palma aceitera en las regiones de Ucayali, Loreto, Huánuco y San Martín, de las cuales 4109493 ha son áreas con bosque y 498567 ha son zonas sin bosque o áreas degradadas, lo cual al comparar estas cifras con el resultado total de áreas de palma aceitera en este estudio, para el año 2020 (95 048,37 ha), representan solamente el 2,06\% de área aprovechada para este cultivo. Siendo para la región Ucayali el 2,29\% de 1927425 ha de área óptima para aprovechar, 13,91\% de 252766 ha para San Martín, 0,64\% de 2118799 ha para Loreto y 0,73\% de 309070 ha para Huánuco. 
La BBC (2012), posiciona al cultivo de palma aceitera como uno de los principales drivers de la deforestación de la Amazonía peruana. Mientras que, los estudios publicados por McCarthy (2020), sostienen que la mayor pérdida de cobertura forestal desde el 2001 hasta el 2019 se dio en el departamento de Ucayali con aproximadamente 572000 ha, sin embargo, el presente estudio obtiene como resultado que solo el $7,65 \%$ (43 809,94 ha) es palma aceitera al año 2020 , encontrando también que la mayor tasa de deforestación fue en el año 2018 con 35170 ha para este departamento, si bien es cierto, coincide con la mayor tasa de producción e incremento del cultivo de palma, es importante mencionar que el incremento para este año fue 2606,23 ha que equivale al $7,41 \%$ en donde se evidencia que el principal motivo de pérdida de cobertura forestal se puede atribuir a la agricultura migratoria y a la ganadería.

Vijay et al. (2018) menciona que los cultivos de palma aceitera irán aumentando gradualmente al pasar de los años, esto se ve reflejado en sus estudios de los años 2000 - 2005 con un incremento del $1,4 \%$ mientras que entre los años 2006-2015 fue del $5 \%$, se puede observar un continuo aumento del cultivo de palma aceitera a lo largo de los años 2016 2020 en los departamentos de Ucayali, Loreto, San Martín y Huánuco debido a la aprobación de la Resolución Ministerial "Plan Nacional de Desarrollo Sostenible de Palma Aceitera 2016 - 2025" (MINAGRI, 2016), lo cual a su vez se puede sustentar con el resultado obtenido para el incremento del cultivo entre los años 2016-2020 que es del 11\%, mientras que un estudio realizado por Dammert (2015) sobre el aumento de la palma aceitera en el Perú, indica que para el año 2021 se espera alcanzar las 250000 ha de palma aceitera en el Perú lo cual no es factible debido a la baja tasa de incremento porcentual de palma aceitera como se demuestra en el estudio ya que se estaría hablando de un incremento de más del $100 \%$ para el año 2021.

Para el año 2018 se obtuvo como resultado 91357,80 ha de palma aceitera difiriendo de esta forma con el estudio realizado por el MAAP (2018) con un resultado de 86600 ha , esta diferencia se debe al tiempo de la evaluación de los datos ya que en el presente estudio se tomó una línea temporal más amplia y en la evaluación de imágenes empleada para cada estudio siendo Planet NICFI y Digital Globe respectivamente, no obstante, SPDE y JUNPALMA para el año 2017 obtuvieron 88 559, 04 ha y 88620 ha respectivamente, al compararlo con el resultado obtenido en este estudio, de $86 \quad 866,18$ ha se evidencia una variación del $2,25 \%$ aproximadamente.

Según MINAGRI (2020), citado por Escobedo (2021), para el año 2019 se encuentran 108020 ha instaladas de cultivo de palma aceitera, mientras que en el presente estudio para el mismo año se determinó 94 656,40 ha presentado una variación de 13 363 ha aproximadamente, esto se puede deber a que MINAGRI trabaja con información primaria actualizada y la interpreta como áreas legalmente 
cultivadas ya sean en fases tempranas como áreas recién instaladas de palma aceitera, dificultando de esta forma la interpretación visual originando así estos grandes sesgos en los resultados.

\section{CONCLUSIONES}

Se estimó que la superficie total del cultivo de palma aceitera (Elaeis guineensis Jacq.) en el área de estudio para el año 2020 resulta en 95 048,37 hectáreas, encontrándose un incremento total de 9 925,42 ha desde el año 2016. Asimismo, se encontraron 16 plantas procesadoras de aceite de palma aceitera y se generó una aplicación en Google Earth Engine para la visualización de esta información.

\section{AGRADECIMIENTOS}

Agradecemos profundamente a SPDE Sociedad Peruana de Ecodesarrollo pues este trabajo se desarrolló en el marco del proyecto "La Revolución de la Cero Deforestación”, con financiamiento de The National Wildlife Federation (NWF) y Norwegian Agency for Development Cooperation (NORAD). También agradecemos a la Junta Nacional de Palma Aceitera del Perú (JUNPALMA), a Víctor Galarreta y a Richard Vaca de Earthworm Foundation por la información otorgada.

\section{LITERATURA CITADA}

Angulo, D., Beltrán, E., Murillo, M., Valdizan, M. (2017). Producción y Comercialización de derivados oleaginosos de Palma Aceitera. [Tesis de Maestría, Universidad Peruana de Ciencias Aplicadas (UPC)]. https:// repositorioacademico.upc.edu.pe/ handle/10757/621632

BBC (03 de mayo del 2012). Palma Aceitera, "Agente de Deforestación en Perú". BBC Mundo. https:// www.bbc.com/mundo/ noticias/2012/05/120503_peru_palm a_aceitera_am

Butler, R., Laurance, W. (2009). Is oil palm the next emerging threat to the Amazon?. Tropical Conservation Science, 2 (1), 1-10. https:// journals.sagepub.com/doi/

full/10.1177/194008290900200102

Dammert, L. (2014). Cambio de uso de suelos por Agricultura a gran escala en la Amazonía Andina: El caso de la Palma Aceitera. Iniciativa para la Conservación en la Amazonía Andina - ICAA. https:// www.actualidadambiental.pe/wpcontent/uploads/2014/09/Cambiode-uso-de-suelo-y-el-caso-de-lapalma-aceitera-en-la-Amazon\%C3\% ADa.pdf

Dammert, L. (2015). Hacia una Ecología Política de la Palma Aceitera en el Perú. OXFAM. https:// oi-files-cng-prod.s3.amazonaws.com/ peru.oxfam.org/s3fs-public/ file_attachments/Hacia\%20una\% 20Ecolog\%C3\%ADa\%20Pol\%C3\%ADtica\% 20de\%20la\%20Palma\%20Aceitera\%20en \%20el\%20Per\%C3\%BA.pdf 
O. Yupanqui, J. Julca y E. Valerio: Estimación de la superficie cultivada de Palma Aceitera mediante interpretación visual de imágenes satelitales durante los años 2016 - 2020

Escobedo, A. (2021). Agronegocios y Crisis Climática en el Perú. Monocultivos, Deforestación y Emisiones de Carbono en la Amazonía Peruana. Oxfam América Inc. https:// peru.oxfam.org/latest/policy-paper/ agronegocios-y-crisis-climatica-en-peru

Gorelick, N., Hancher, M., Dixon, M., Ilyushchenko, S., Thau, D., Moore, R. (2017). Google Earth Engine: Planetary-scale geospatial analysis for everyone. Remote Sensing of Environment, 202, 18-27. https:// doi.org/10.1016/j.rse.2017.06.031

Green, Edmund P., et al. "Remote sensing techniques for mangrove mapping." International journal of remote sensing 19.5 (1998): 935-956. https: / /

doi.org/10.1080/014311698215801

Kongsager, R., Reenberg, A. (2012). Contemporary land-use transitions: the global oil palm expansion (Reporte $\mathrm{n}^{\circ}$ 4). Global Land Project. https:// orbit.dtu.dk/en/publications/ contemporary-land-use-transitionsthe-global-oil-palm-expansion

Leveau, R. (2018). Sustentabilidad de fincas productoras de Palma Aceitera (Elaeis guineensis), en el valle del río Shanusi, Loreto. [Tesis de Maestría, Universidad Nacional Agraria La Molina]. https://

repositorio.lamolina.edu.pe/

bitstream/handle/UNALM/3810/

leveau-tuanama-roberto.pdf?

sequence $=1$ \&isAllowed $=\mathrm{y}$

MAAP (12 de noviembre del 2018). Línea base de palma aceitera para la Amazonía Peruana. Monitoring of the Andean Amazon Project. https:// maaproject.org/2018/palma-aceiteraperu/
McCarthy, N. (2020). A Spotlight on Exponential Peruvian Palm Oil Growth: ESG Risks for Cargill Subsidiary, Cargill Americas Peru SPL. Climate Advisers Trust. https:// climateadvisers.org/wp-content/ uploads/2020/12/Climate-Advisers_ASpotlight-on-Exponential-PeruvianPalm-Oil-Growth-2020.pdf

MINAGRI (Ministerio de Agricultura y Riego del Perú) (2012). Estudio sobre la potencialidad de la Palma Aceitera para reducir la dependencia de Oleaginosas importadas en el Perú. Dirección de Información Agraria, Dirección General de Competitividad Agraria. http: / / palma.webcindario.com/ potencialidadpalma.pdf

MINAGRI (Ministerio de Agricultura y Riego del Perú) (2016). Plan Nacional de Desarrollo Sostenible de la Palma Aceitera en el Perú 2016-2025. https: / /www.minagri.gob.pe/portal/ download/pdf/marcolegal/ normaslegales/ resolucionesministeriales/2016/junio/ rm281-2016-minagri.pdf

MINAGRI (Ministerio de Agricultura y Riego) (2020). Estadísticas de extensiones (ha.) de palma aceitera instalada y cosechada, para los años 2000 y 2019 . Ministerio de Agricultura y Riego.

OXFAM (Comité de Oxford para el alivio del hambre) (2017). El aumento de cultivos de palma aceitera amenaza la Amazonía Peruana. OXFAM International. https: / / www.oxfam.org/es/el-aumento-decultivos-de-palma-aceitera-amenazala-amazonia-peruana 
Planet. (23 de mayo del 2021). NICFI Data Program User Guides. Planet. https://assets.planet.com/docs/ NICFI_UserGuidesFAQ.pdf

Planet. (23 de mayo del 2021). Planet Basemaps for NICFI Data Program Addendum to Basemaps Product Specification. Planet. https:// assets. planet.com/docs/

NICFI_Basemap_Spec_Addendum.pdf

QGIS.org (2021). QGIS A Free and Open Source Geographic Information System. QGIS Association. http://www.qgis.org

Rubín de Celis, E. (2020). Práctica 1: Interpretación Visual de Imágenes de Satélite. Curso: Teledetección Forestal, UNALM. http:// www.lamolina.edu.pe/Facultad/ forestales/teledeteccion/IMAGES/ Practicas/Pr\%C3\%A1ctica\%201_2020.pdf

Sáenz, G. (2017). Agroindustria de la Palma Aceitera: Alternativa sostenible que promueve desarrollo socioeconómico en la Amazonia. JUNPALMA PERÚ. https:// junpalmaperu.org/sites/default/files/ archivos/2017/publicacion/05/ agroindustria-de-la-palma-aceitera-enperu-enero2017.pdf

SPDE. (2019). Camino hacia la libre Deforestación en la cadena de Palma Aceitera del Perú. Sociedad Peruana de Ecodesarrollo. https: / / sociedadsostenible.co/wp-content/ uploads/2020/02/Policy_Camino-haciala-libre-deforestacion_SPDE_NWF_CDise \%C3\%B102.pdf
Svatonova, H. (2016). Analysis of Visual Interpretation of Satellite Data. International Archives of the Photogrammetry, Remote Sensing \& Spatial Information Sciences, 2(16), 675-681. https://doi.org/10.5194/ isprs-archives-XLI-B2-675-2016

Vijay, V., Reid, C., Finer, M., Jenkins, C., Pimm, S. (2018). Deforestation risks posed by oil palm expansion in the Peruvian Amazon. Environmental Research Letters, 13(11), 114010. https://doi.org/10.1088/1748-9326/ aae540 